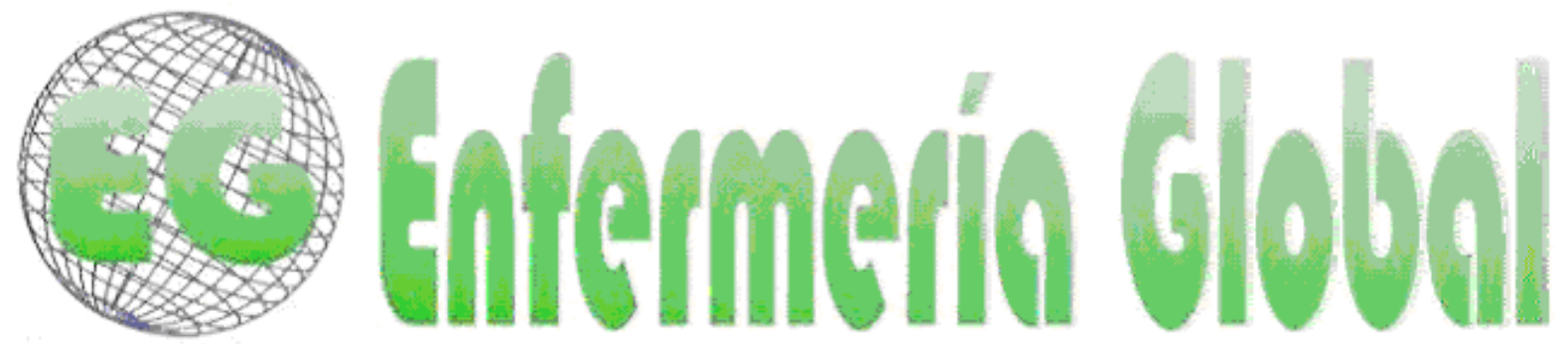

\title{
Significados de la vinculación universidad-comunidad para los actores sociales involucrados en la cátedra Enfermería Comunitaria del Decanato de Ciencias de la Salud en la Universidad Centroccidental "Lisandro Alvarado"
}

Meanings of the university links-community for actors involved in community nursing chair of the dean of science university health Centrocidental "Lisandro Alvarado"

\section{${ }^{*}$ Camejo Giménez, Rafael Alejandro **Rebolledo Malpica, Dinora Margarita}

*Magíster en Educación. Lcdo. en Enfermería. Docente Programa de Enfermería de la UCLA. E-mail: rafael.camejo@hotmail.com **Doctora en Enfermería por la Universidad de Alicante. Magister en Educación. Docente Programa de Enfermería de la UCLA. Venezuela.

Palabras clave: enfermería; comunidad; universidad; fenomenología

Keywords: Nursing; community; college: phenomenology.

\section{RESUMEN}

El presente estudio tuvo como objetivo general conocer los significados de la vinculación UniversidadComunidad para los actores sociales involucrados con la Cátedra Enfermería Comunitaria del Decanato de Ciencias de la Salud en la Universidad Centroccidental "Lisandro Alvarado." Tuvo un enfoque fenomenológico, basándose en los referentes teóricos del Interaccionismo Simbólico, Estructuralismo Dialéctico y el Existencialismo de Husserl. Se seleccionaron nueve (09) actores sociales, tres (03) docentes, tres (03) estudiantes del octavo semestre y tres (03) miembros del comité de salud de la comunidad de Las Tunas en el pueblo de Tamaca. Se utilizó la técnica de la entrevista abierta para obtener la información de la que emergieron los significados mediante la interpretación. Los hallazgos mostraron que: 1) Para los docentes existe un significado claro y definido de Enfermería Comunitaria y de lo que se espera de los estudiantes. 2) Para los estudiantes, a través de los significados que le dieron a sus propias experiencias, desempeñan un rol dinámico, sin embargo, muchos de ellos expresaron que no tienen vocación para el desempeño de trabajos comunitarios, a pesar de poseer los conocimientos en este campo. 3) Para los miembros de la comunidad mediante el significado que le dieron a su experiencia se evidenció que existen cambios importantes en el desarrollo de las actividades, pero afirmaron que se deben fortalecer los valores. Como reflexiones finales se reveló que existe una vinculación entre la universidad y la comunidad la cual es débil en algunos aspectos, como recursos, perfil comunitario y vocación del estudiante.

\section{ABSTRACT}

This study aimed to generally know the meanings of the University-Community link to social actors involved with the Department of Community Nursing Dean of Health Sciences at the University "Lisandro Alvarado." It took a phenomenological approach based on the references Symbolic 
Interaction theory, structuralism and existentialism Dialectic of Husserl. We selected nine (09) social actors, three (03) teachers, three (03) students of the eighth semester and three (03) members of the health committee of the community of Las Tunas in the village of Tamaca. We used open interview technique to get the information from which emerged the meanings through interpretation. The findings showed that: 1) for teachers there is a clear and definite meaning of Community Nursing and what is expected of students. 2) For the students through the meanings they gave to their own experiences play a dynamic role. However, many of them expressed that they have no vocation for the performance of community service, despite having knowledge in this field and finally 3 ) for members of the community through the meaning they gave to their experience showed that there are significant changes in the development of activities, but they stated that the values should be strengthened. As final reflections it was revealed that there is a link between the university and the community which is weak in some aspects such as resources, community profile and vocation of the student.

\section{INTRODUCCIÓN}

Es de hacer resaltar que las instituciones de educación superior y las comunidades, de acuerdo con Watson y otros ${ }^{(1)}$ aprenden unas de las otras en un proceso de colaboración, impulsando nuevas maneras de trabajar juntas, de crear conocimientos nuevos y de afrontar problemáticas sociales complejas, como la pobreza, la educación, la salud, el bienestar, la seguridad, la violencia, la sostenibilidad medioambiental y la disponibilidad de agua y alimentos. Problemas que afectan tanto a las poblaciones desposeídas, como al grueso de la sociedad.

Destaca el autor mencionado que un aspecto fundamental de esta colaboración se relaciona con las estructuras actuales entre la comunidad y la universidad, a fin de incentivar, dinamizar e impulsar la acción investigadora con compromiso comunitario entre académicos y expertos de las comunidades. Los investigadores universitarios impulsan el trabajo con grupos y organizaciones comunitarias para fomentar el cambio de mentalidad colectivo que abandone la transferencia de ideas a favor del intercambio de conocimientos.

Según el autor los cambios significativos deben emerger del Estado bajo una óptica social, por eso los actores tienden a involucrarse de manera activa y no pasiva en un verdadero proceso de transformación cultural, el cual busca nuevos modelos paradigmáticos para que las clases desposeídas se beneficien. Asimismo Hall (2) infiere que:

...en el caso de las comunidades que cuentan con instituciones de educación superior, los recursos colectivos de estas universidades y centros de formación (estudiantes, personal académico, instalaciones, fondos para la investigación, capacidad investigadora y recursos didácticos) constituyen el activo más rico, accesible e infrautilizado para hacer realidad la aspiración de cambio social y sostenibilidad... (p. 13).

El autor acota la importancia que representan las instituciones educativas a nivel universitario en el progreso de las comunidades, ya que su vinculación proporciona un desarrollo sustentable en las mismas. Aunque la realidad evidencia, conforme a las ideas citadas, una inadecuada utilización de los recursos materiales y humanos disponibles en estas casas de estudio a favor de la sociedad en general.

Destacan Rivas y otros (3) que las universidades venezolanas, no sólo son reconocidas como instituciones que ofertan educación e investigación, sino también como protagonistas del desarrollo local y regional al interactuar con los gobiernos 
locales y regionales y con las comunidades como socios claves en el crecimiento y en el desarrollo económico de un determinado territorio.

Es por ello que la universidad constituye un modelo por medio del cual el individuo descubre un conjunto de capacidades que lo preparan para evolucionar psicosocialmente y alcanzar el máximo desarrollo dentro de los escenarios que se presentan en una sociedad cambiante. En este sentido, la Ley del Servicio Comunitario ${ }^{(4)}$ plantea como una herramienta obligatoria del estudiante de pregrado de educación superior proyectar los conocimientos adquiridos dentro de la universidad en la comunidad bajo una realidad percibida y tangible.

Claramente se observa cómo el Estado venezolano intenta implementar un instrumento legal que articule de una manera integral los diferentes saberes que se originan en la universidad, con el objetivo de crear un beneficio social a través del mejoramiento de las comunidades, para lo cual involucra a los estudiantes de las diferentes carreras universitarias. De igual forma, las universidades poseen programas con las que intentan vincularse con la comunidad, incluso mucho antes de la prescripción legal comunitaria. Tal es el caso de la Universidad Centroccidental Lisandro Alvarado (UCLA) ubicada en Barquisimeto Estado Lara, que posee una Dirección de Extensión integrado por profesionales miembros de la comunidad universitaria, quienes manejan un conjunto de estrategias y políticas que permiten y coadyuvan el desarrollo de la región.

Se observa cómo la UCLA gestiona llevar el conocimiento que produce a la sociedad, específicamente a las comunidades, para fomentar su crecimiento. Entre sus programas de Educación Superior se encuentra el desarrollado desde la Licenciatura en Enfermería del Decanato de Ciencias de la Salud en la cátedra Enfermería Comunitaria, Cátedra que se dicta en el sexto semestre de la carrera y pertenece a las asignaturas del Eje Curricular Comunitario, enmarcada en el área de formación profesional cuyo propósito es la formación de profesionales con una concepción holística del individuo y su grupo familiar para comprender, actuar e involucrarse en situaciones reales y concretas de salud de la comunidad.

Por ello, el docente debe trasladarse a las comunidades con la finalidad de supervisar el trabajo de sus estudiantes y estos últimos desempeñar actividades de la enfermería comunitaria. Es por ello, que debe existir una articulación entre el docente estudiante - comunidad, aunque existen ciertas dificultades que no permiten la fluidez del trabajo como lo expresa el actor social docente 1:

- "...muchas veces los estudiantes se desmotivan porque no ven la participación...de la comunidad."

Ante la situación planteada, esta desmotivación se puede atribuir a la falta de compromiso por parte de los actores sociales involucrados, abandonando así la necesidad real del conglomerado (comunidad). Este contexto ha sido evidenciado en las experiencias vividas por el investigador tanto es su rol como estudiante como en su rol de docente comunitario.

Por otro lado, se origina una desconexión de los docentes con los problemas de la comunidad, escasa evaluación del comportamiento de los estudiantes dentro de la práctica comunitaria, falta de comunicación para informar inquietudes y propuestas 
por parte de los actores sociales y alejamiento del vínculo universidad-comunidad. Esto se refleja en lo expuesto por el actor docente 1:

-“...considero que no contamos con una cultura preventiva sino curativa..."

No obstante, es conocido por todos los actores involucrados en el estudio, que el perfil de enfermería es netamente comunitario y por ende preventivo, siendo esto último lo que se espera alcanzar con la vinculación Universidad-Comunidad. Asimismo, los docentes realizan las observaciones aisladas y desvinculadas de una realidad comunitaria. Esto trae gran inquietud en la comunidad y estudiantes en relación con el desenvolvimiento como profesionales de dicha área, puesto que la acción comunitaria es relevante para la satisfacción de las necesidades de la población.

Bajo esta perspectiva se evidencia que no se da continuidad a los proyectos comunitarios iniciados por intervenciones anteriores de la Universidad, según los actores sociales estudiantiles, por falta de recursos para desarrollar las actividades, así lo expresa el actor social 4:

- “... no contamos con transporte y seguridad para ir a la comunidad..."

Ante lo descrito, surgen las siguientes interrogantes: ¿Cuáles son los significados de la vinculación Universidad-Comunidad para los actores sociales involucrados en la Cátedra Enfermería Comunitaria del Decanato de Ciencias en la Salud en la Universidad Centroccidental "Lisandro Alvarado"? ¿Cómo es el vínculo Universidad Comunidad desde la Cátedra Enfermería Comunitaria? ¿Cómo son los factores que influyen en el vínculo Universidad - Comunidad desde la percepción de los actores sociales involucrados? ¿Cuáles son los significados de los actores sociales, en la relación Universidad, Docente, Estudiante, Comunidad? Estas interrogantes permitieron indagar sobre los significados de la vinculación universidad-comunidad para los actores sociales involucrados en la cátedra Enfermería Comunitaria del Decanato de Ciencias de la Salud de la Universidad Centroccidental "Lisandro Alvarado."

\section{OBJETIVOS DE LA INVESTIGACIÓN}

Conocer los significados de la vinculación Universidad-Comunidad para los actores sociales involucrados en la Cátedra Enfermería Comunitaria del Decanato de Ciencias en la Salud en la Universidad Centroccidental "Lisandro Alvarado".

\section{METODOLOGÍA}

Esta investigación se abordó bajo el paradigma emergente, con un enfoque eminentemente cualitativo, sustentado en la descripción de hechos observables en el contexto global donde se desarrolla. Al respecto, Morse ${ }^{(5)}$ manifiesta "La investigación cualitativa es la herramienta por excelencia para la construcción de la enfermería como profesión, disciplina y ciencia". Esto hace que la investigación cualitativa se convierta en la herramienta por excelencia de la indagación humanística y por ende de la investigación en enfermería, buscando al ser como parte única de la realidad que emerge. 
Se interpreta que la esencia del paradigma interpretativo es a través del desarrollo de un sistema de relaciones, es comprender los significados de la vinculación universidad-comunidad para los actores sociales involucrados con ciertos criterios de orden teórico que llevan al investigador a la confrontación de la realidad, la cual se va enriqueciendo en la medida que se va apropiando del uso crítico de la teoría y amplía sus horizontes referenciales.

En relación con la fenomenología ${ }^{(6)}$ se aspira al conocimiento estricto de los fenómenos. No entiende así los fenómenos, pues para esta corriente filosófica los fenómenos son, simplemente, las cosas tal y como se muestran, tal y como se ofrecen a la conciencia. Un estudio fenomenológico se describe ${ }^{3}$ como el significado de las experiencias vividas por una persona o grupo de personas acerca de un concepto o fenómeno, la fenomenología no está en última instancia interesada en la interpretación. La cuestión típica formulada no es "¿qué causa X?', sino "¿qué es X?' Se preocupa por los aspectos esenciales de tipos de experiencia o conciencia.

De lo expuesto se denota que, en esta investigación se trabajó con los fenómenos suscitados en la comunidad donde los informantes claves realizaron sus aportes en el área de enfermería comunitaria a través del proceso de entrevistas abiertas cualitativas y donde el investigador tuvo la oportunidad de interactuar de manera dialógica con el entrevistado, dándole sentido a la información de acuerdo a los parámetros descritos a continuación.

\section{Actores Sociales}

Ruiz Olabuenaga (7) menciona que son un grupo de personas que aportaron información, sin que necesariamente sea representativo el universo o población que se estudia. La selección de los actores sociales se realizó de acuerdo con los supuestos teóricos de la investigación cualitativa, El escenario de estudio fue la comunidad de Las Tunas en el pueblo de Tamaca.

Es de hacer resaltar que los actores se seleccionaron porque están involucrados directamente en la enfermería comunitaria, es por ello que el criterio de selección para este estudio estuvo focalizado en cada participante involucrado, del cual se seleccionaran tres (03) docentes que imparten la asignatura Enfermería Comunitaria, docentes de amplia trayectoria en este campo y que se denominan en esta investigación (actor social Docente 1, 2 y 3), tres (03) estudiantes del octavo semestre que cursaron esta asignatura, que poseen todas las vivencias de enfermería comunitaria a lo largo de la carrera, los cuales se denominan en esta investigación (actor social estudiante 4,5 y 6 ) y tres (03) miembros del concejo comunal de las comunidad donde la universidad realiza sus prácticas comunitarias, líderes comunitarios protagonistas en su espacio, que en este estudio se denominan (actor social comunitario 7, 8 y 9); dando un total de nueve (09) actores sociales, a los cuales se les entregó personalmente una carta solicitando su autorización para participar en este estudio, a través de la negociación se determinó el lugar y la hora para realizar las entrevistas quienes aportaron la información necesaria que llevó al investigador a descubrir los significados que le dan a la vinculación de la Universidad y la Comunidad a través de esta cátedra. 


\section{Recolección de Información}

Para esta investigación se utilizó la entrevista cualitativa abierta propuesta por Tesch citado por Valles ${ }^{(8)}$ ya que es una entrevista personal, directa y no estructurada en la que el investigador hace una indagación exhaustiva para lograr que el entrevistado hable libremente y exprese en forma detallada sus motivaciones, creencias y sentimientos sobre un tema. Es una técnica de intensa interacción personal que posibilita un gran nivel de persuasión y armonía con el entrevistado, dando lugar a una relación sustentada en un clima de confianza, en la cual fluye la confidencia e inclusive la información que normalmente se oculta o no se comparte con nadie, es decir, busca que el entrevistado hable sinceramente de lo que cree de sí mismo.

Para la obtención de la información se aplicó la entrevista abierta de manera individual a los nueve (9) informantes claves involucrados en la asignatura enfermería comunitaria, 01 por cada actor social distribuido de la siguiente manera: (a) Docentes de la asignatura Enfermería Comunitaria. (b) Estudiantes del octavo semestre de enfermería. (c) Miembros del Comité de Salud de la comunidad donde la universidad participa en enfermería comunitaria.

\section{Consideraciones Éticas}

Paris ${ }^{(9)}$ expresó que las consideraciones éticas a tener en cuenta en este tipo de estudio se pueden definir mediante características esenciales que debe cumplir toda investigación de carácter cualitativo y que se resumen y analizan de la siguiente forma, según lo explicado por el autor:

1.Los consentimientos informados: su principio básico es la trasparencia en cuanto a los procedimientos, derechos, deberes de todos los que se involucran en el proceso de la investigación.

2.El anonimato o la confidencialidad, implica la no revelación de informaciones que puedan hacer posible la identificación de los participantes.

En definitiva, la principal consideración ética es el principio de la autenticidad ${ }^{(10)}$. La búsqueda de construcciones individuales o de grupo precisa del conocimiento de los valores, por este motivo la gran responsabilidad ética del investigador se centra en mostrar las construcciones de manera fiel y precisa.

\section{Rigor Científico}

La credibilidad de la información se llevó a cabo mediante la triangulación, ya que es una estrategia convergente dirigida a la comparación e integración de diferentes resultados investigativos. Es conveniente concebirla envolviendo variedades de datos, investigadores y teorías, así como de metodologías en un mismo fenómeno.

Por su parte, Eyssautier de la Mora (11), plantea que en la medida en que la triangulación conduce a una visión más global puede dar lugar a un sesgo hacia la generalización excesiva. De hecho, los resultados obtenidos tratan de organizarse en una teoría útil que estará orientada a su explicación y difusión. 


\section{RESULTADOS}

\section{Discusión y Análisis de la Información}

El proceso de organización y análisis de la información se realizó a través de un proceso de categorización. Para la presentación de los resultados, se utilizaron cuadros numerados uno (01) y dos (02) en los cuales se registra la sistematización de la evidencia, es decir, las voces de los actores sociales y de la relación dialógica con el investigador, llegando a ser una estructura teórica.

Es así como se origina el tema central, siendo la conceptualización de las relaciones reflexivas entre los códigos, subcategorías y categorías que le dan un significado a la realidad contextual de los actores sociales para este estudio. Para comprender el proceso de categorización se define (13) "...una categoría queda definida por un constructo mental...". La categoría de Enfermería Comunitaria y Vinculación Universidad - Comunidad se convierte así en la conceptualización personal a la que llega el investigador y es el tema central de los cuadros 01 y 02 respectivamente, que se presentarán posteriormente.

Es así como la Enfermería Comunitaria queda definida según la interpretación del investigador desde las voces de los actores sociales, centrada en un perfil basado en competencias en el área comunitaria cuya formación técnica y científica garantizan valores que generen la satisfacción de las necesidades comunitarias de los contextos sociales en los que se desempeñe. Esta categoría se ve descrita en el cuadro número uno (01).

Asimismo, la Vinculación Universidad - Comunidad se presenta desde la integración de las voces de los actores sociales y el investigador como una realidad social, que toma en cuenta a la familia y a la comunidad, orientada a la transformación de un contexto que emerge de la interacción permanente y continua entre la universidad y la comunidad a fin de solucionar sus necesidades y conflictos a través de la participación y manejo de recursos humanos y tecnológicos, la cual eleva el nivel social en materia de salud de la población. Esta categoría se ve descrita en el cuadro número dos (02).

Entre tanto, los cuadros uno (01) y dos (02) que representan la estructuración teórica de las categorías descritas quedaron compuestos por cuatro columnas. La primera columna refleja las categorías per se, basadas en las relaciones dialógicas identificadas en las categorías previas del análisis, llamado en este cuadro sub categorías.

En la segunda columna se describe las subcategoría que es la sistematización de la evidencia basada en las relaciones y la interpretación de los códigos. Según Strauss y Corbin ${ }^{(14)}$ la subcategorías son conceptos que pertenecen a una categoría y que le dan claridad adicional y especificidad.

La tercera columna presenta la descripción que no es más que textos puntuales acerca de la temática que emerge desde las voces de los actores sociales y que le da forma a la sub categoría. La descripción "...nos permite dar detalles acerca de la temática referida a la cotidianidad del actor social, con sus propias palabras..." (15) Esto quiere decir, que son textos cortos identificados por el investigador a medida que las entrevistas cualitativas abiertas se iban desarrollando. 
Por último, en la cuarta columna, se presentan los códigos, que son extractos específicos de los textos que reflejan la realidad de los actores desde su propia voz. Según Bustingorry, Sánchez e lbáñez (16) “...los códigos son una asignación o unidad indicativa que determinan la marca concreta que lo incluye dentro de una categoría quedando etiquetada la realidad contextual de los actores sociales..." Estos no son más que extractos puntuales que el investigador consideraba pertinentes durante la transcripción en relación con la investigación y al cual le asigna una nomenclatura que los identifica.

\section{Cuadro 01. Tema Central: Enfermería Comunitaria}

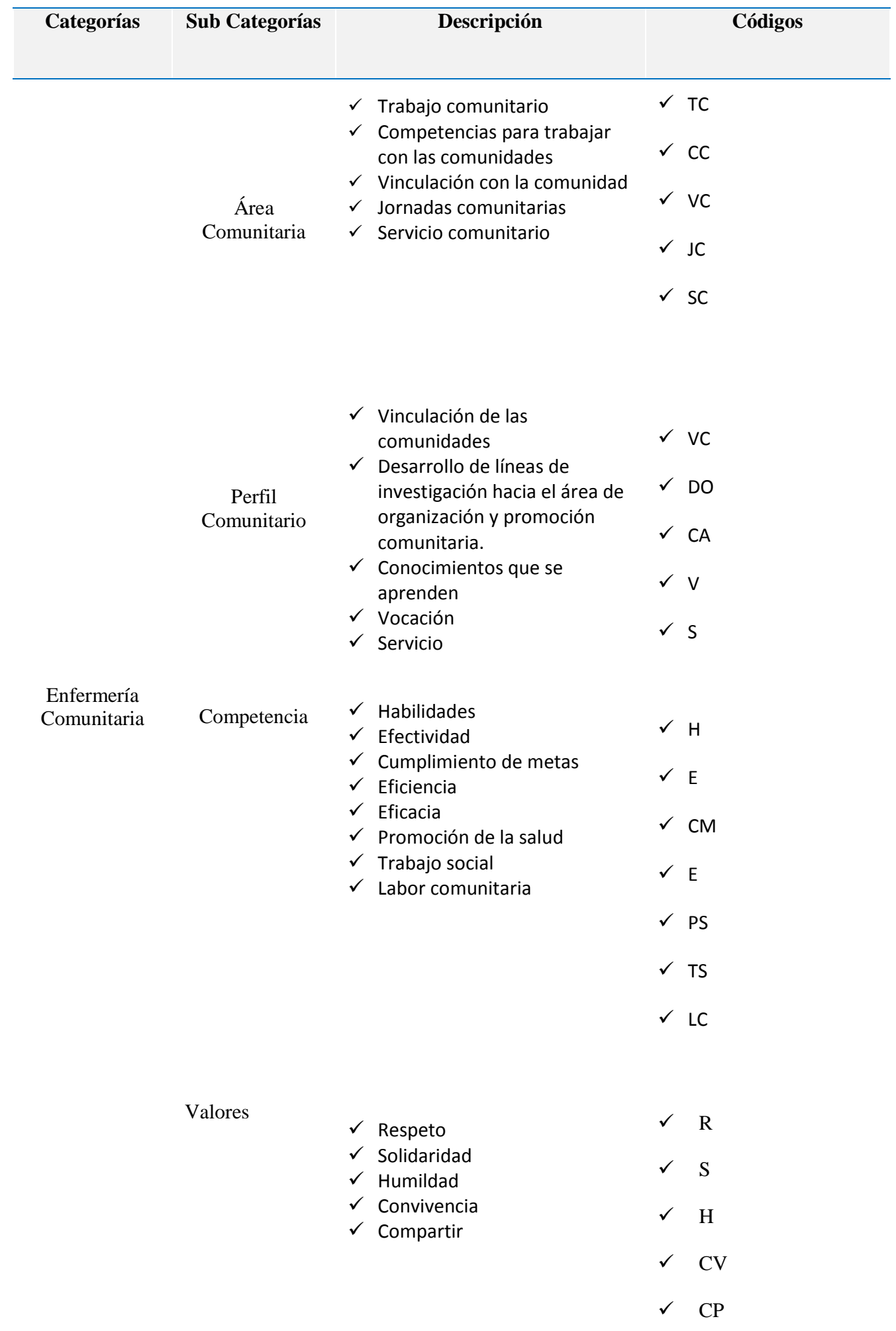




\begin{tabular}{lllll} 
& $\checkmark$ & Proceso de reingeniería & & \\
& $\checkmark$ & Conocimiento & $\checkmark$ & PR \\
\multirow{4}{*}{ Formación } & $\checkmark$ & Técnicas & & \\
& $\checkmark$ & Estrategias & $\checkmark$ & C \\
& & & & \\
& & & T \\
& & & ET
\end{tabular}

Fuente: El autor (2012)

\section{Cuadro 02 Tema Central: Vinculación Universidad - Comunidad}

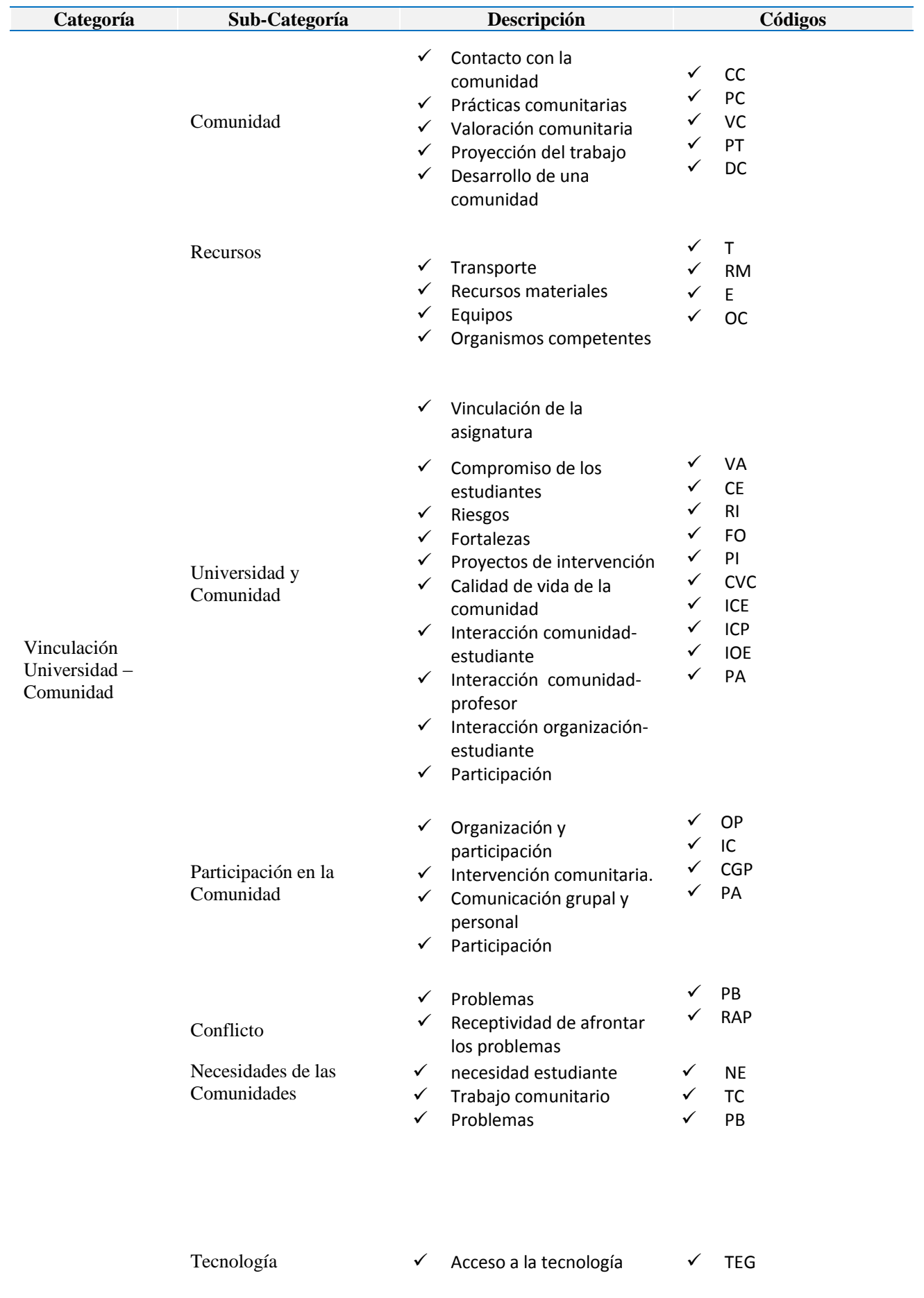

Fuente: El autor (2012) 


\section{Hallazgos}

\section{Significados que los Actores Sociales le Otorgan al Área Comunitaria desde las Actividades de Enfermería Comunitaria.}

Los significados que los Actores Sociales le otorgan al Área Comunitaria tienen estrecha relación con el concepto de Enfermería Comunitaria y las actividades que allí se desarrollan. Es por ello, que la enfermería comunitaria se concibe como un espacio social para la reflexión - intervención sobre la práctica, basado en un trabajo comunitario así como lo expresó el actor social Docente 1

- "Tengo 12 años trabajando como docente en el área comunitaria y 25 años de experiencia en el trabajo comunitario"

Esta práctica se plantea en el marco del esquema pedagógico que se plantea a partir de una visión estructural de los procesos salud - enfermedad - atención, para la construcción y sistematización de la práctica profesional con competencias personales, laborales y comunitarias, en los diversos ámbitos y unidades de intervención de enfermería en y con la Comunidad donde se brindan jornadas y servicios destinado a los ciudadanos y ciudadanas.

Desde esta, según la óptica de Ramírez ${ }^{(17)}$ plantea que la enfermería comunitaria es la intervención comunitaria en salud que se da dentro de un espacio de desarrollo social. Se trata aquí de hacer énfasis en la identificación y canalización de las necesidades de esa área geográfica utilizada en el concepto, por cuanto ello posibilita una nueva alternativa de abordaje metodológico en los estudios sobre la sociedad, que contribuye a visiones que trasciendan el nivel de los estudios específicos y profundicen en la complejidad de la sociedad.

De lo referido por el autor citado y de la propia experiencia del investigador es posible inferir que el área comunitaria es la intervención social de Enfermería en la satisfacción de las necesidades de salud de un grupo de individuos enmarcados en un mismo espacio territorial que comparten necesidades, intereses particulares y una forma de ver la vida en comunidad. Las comunidades del estudio constituyen la unidad territorial elemental para la atención primaria de salud, bajo la supervisión del Consejo Comunal donde los estudiantes realizan sus prácticas de enfermería.

Asimismo, se puede fijar la posición investigativa que la Enfermería Comunitaria viene a ser la prestación de servicios de enfermería en cualquier ámbito de salud donde se desarrolla un trabajo comunitario basado en las competencias de los actores (docentes, estudiantes y habitantes de la comunidad), desarrollando y ejecutando actividades asistenciales de prevención de la salud a través de jornadas y servicios desde adentro hacia afuera con vinculación comunitaria, como lo expresa el actor social estudiante 5 al manifestar

- "Es importante llevar la información a la comunidad, sobre todo la prevención"

Esto denota la función de la Enfermería Comunitaria desde la perspectiva de cada actor social y su vinculación con la comunidad. 


\section{Fundamento de la Vinculación Universidad - Comunidad como Perfil Comunitario de Enfermería}

El fundamento de la vinculación Universidad - Comunidad como perfil Comunitario de Enfermería busca que la enfermería comunitaria logre en el estudiante la orientación de este proceso, por cuanto se parte de un perfil basado en el trabajo comunitario como fundamento en la práctica de Enfermería, el cual se realiza desde los primeros semestres de la academia como lo manifiesta el actor docente 1

- "El decanato de medicina nació con un perfil comunitario, es decir, el estudiante iba a desarrollar la vinculación con las comunidades desde los primeros semestres de la carrera y por supuesto que actualmente se mantiene este perfil"

Este planteamiento se apoya en líneas de investigación que promueve la organización Universitaria; asimismo permite que el estudiante desarrolle sus conocimientos teóricos y los ponga al servicio de la comunidad, con vocación de servicio, pues, trabajará en el aprendizaje de los componentes del esquema de la práctica desde la comunidad, centrado en el conocimiento aplicado en la atención y su relación con las necesidades de los habitantes que permite abordar las diferentes concepciones, marcos conceptuales y metodológicos planteados en el espacio de la fundamentación comunitaria, desplegando en forma paralela y simultánea las estrategias de intervención planteadas en la práctica profesional, adecuando el predominio de sus componentes a la realidad en la cual está inmerso.

Almaguer plantea ${ }^{(18)}$ al respecto que el Perfil Comunitario de Enfermería constituye un ambicioso fin último de la enfermería reflejado en un documento que intenta captar ideas, aspiraciones y preocupaciones de toda la comunidad que necesita ser contrastada con las características clave de una comunidad sostenible cuya respuesta permitirá valorar al municipio según cada característica como baja, media o alta $(\mathrm{s} / \mathrm{p})$

Así se analiza que es importante la capacidad de los estudiantes que ingresan a una comunidad y las estrategias que desarrollan, esto les permite mejorar y entender el contexto en el que operan los hogares e instituciones locales y poder identificar las necesidades de la comunidad desde los primeros semestres. Así mismo, Almaguer expresa que el perfil de enfermería comunitaria intenta captar ideas para ser distinguidas con las necesidades de la comunidad y así poder tener una valoración integral que permita una acción efectiva.

De igual manera, la Enfermería Comunitaria promueve la salud, basada en el desarrollo del proceso de educación permanente, en sus diferentes ámbitos de intervención con la comunidad, busca vaciar sus conocimientos apoyándose en la investigación, docencia y promoción de la salud, a fin de garantizar un óptimo servicio médico asistencial y de educación para la salud.

\section{Estructura Teórica del Significado de los Hallazgos}

En el presente apartado se organizan los significados otorgados a los hallazgos, presentando una propuesta teórica de los resultados de la interpretación de la información proporcionada por los nueve actores sociales, relacionados con la vinculación Universidad - Comunidad a través de la Cátedra Enfermería Comunitaria del Decanato de Ciencias de la Salud de la Universidad Centroccidental "Lisandro 
Alvarado", lo cual dio lugar a describir cada uno de los hallazgos, agrupándolos posteriormente por tipo de actores. Es decir, un infograma por los docentes, uno por los estudiantes y uno por los miembros de la comunidad. El análisis permitió describir las definiciones de cada uno de ellos asociados a la enfermería. Es así que por cada grupo se señaló con una línea continua color anaranjada a los actores sociales 1, 4 y 7. Así mismo, los actores sociales 2,5 y 8 se señalaron con una línea discontinua color verde. Finalmente los actores sociales 3,6 y 9 se señalaron con una línea punteada azul. A continuación se presenta tres infogramas, uno de los Docentes, otro de los Estudiantes y uno de la Comunidad.

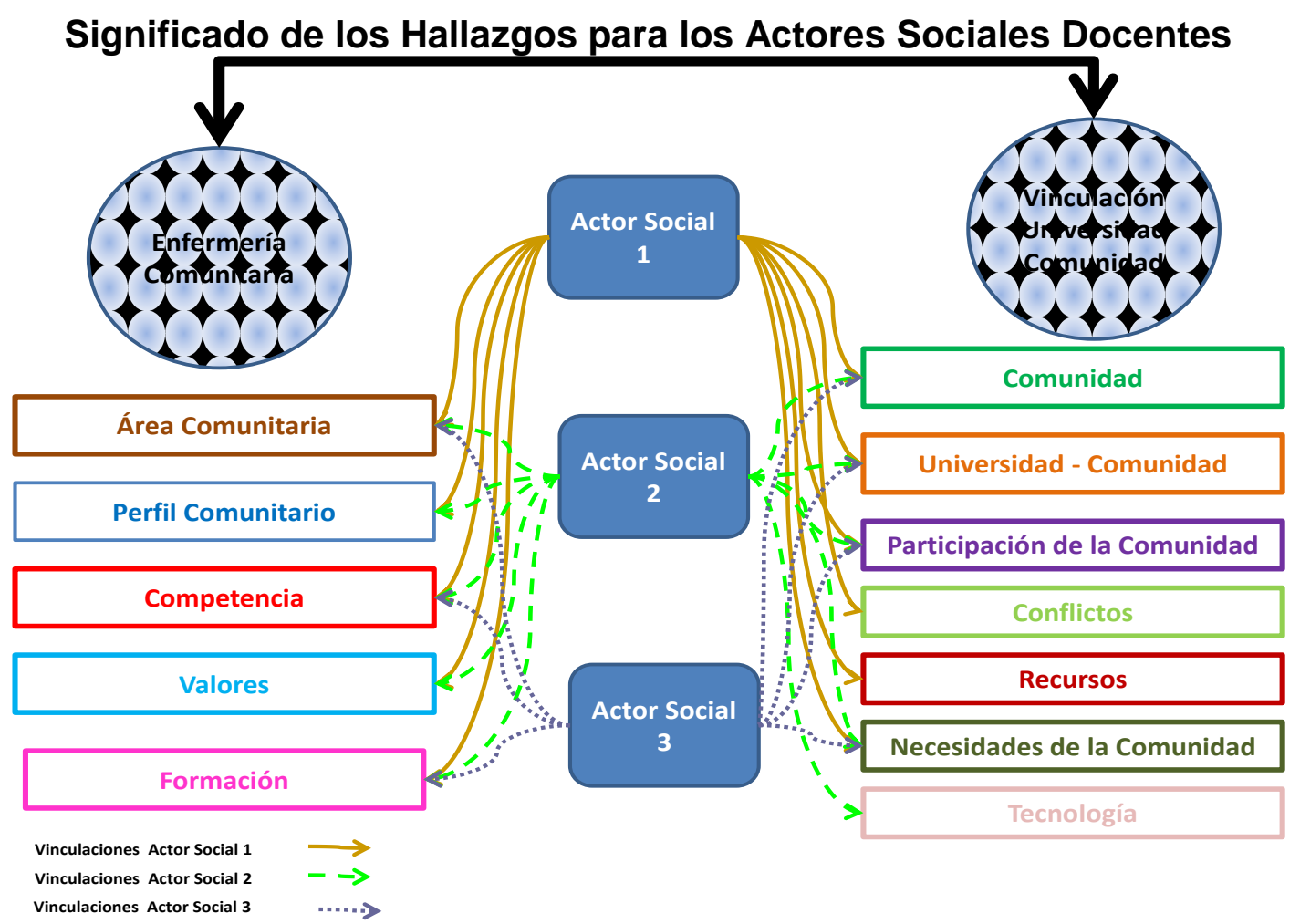

Infograma 1: Vinculación de las Categorías de Análisis con las subcategorías de los Actores Sociales 1, 2, 3

Como se observa en el infogrma 01, se evidencia en los hallazgos suministrados por los docentes que existe una clara y completa definición de lo que es la enfermería comunitaria, que conocen perfectamente hacia donde se dirigen los estudiantes y qué se espera de ellos, así como se evidenció en el actor social Docente 1

- "La idea es desarrollar ciertas competencias en los estudiantes que puedan desarrollarse con la comunidad y aprender no solamente de las habilidades...sino también algunos valores fundamentales como es el respeto, la solidaridad...que aprenden a identificar situaciones problemáticas y resolverlas"

Igualmente se evidenció que dentro de este marco comunitario, la enseñanza universitaria debe ser pertinente en el contexto de las necesidades sociales, económicas, ambientales y culturales de las diferentes comunidades donde el estudiante realiza su práctica de enfermería, de manera de otorgarle a las 
comunidades mayor capacidad de respuesta a las dificultades generales en materia de salud, donde los estudiantes se valoren a sí mismos, a los docentes de la institución y a los miembros de la comunidad.

Otro aspecto que se observó en cada uno de los hallazgos presentados, es el conocimiento generado y transmitido a través de la práctica comunitaria, ya que encuentra su desarrollo pleno cuando se realizan actividades de asistencia en la comunidad del cual depende la articulación entre el conocimiento obtenido en la Universidad y las distintas necesidades de la comunidad. De esta forma se vio expresado por el actor social Docente 3

- "El trabajo comunitario...es el acercamiento social que tenemos con el individuo, con la familia...es el cuidado de la persona, la comunidad y se brinda a través de la repuesta humana y dirigida totalmente a la promoción de la salud"

Se constató que, a través de la práctica profesional, el docente tiene la capacidad de trasmitir sus conocimiento a los estudiantes para así generar patrones de conducta adecuados inmersos en la práctica comunitaria.

Asimismo, se afirma que la formación, integración y calidad que debe encarar la Universidad y la Comunidad en relación con los procesos sociales, productivos, económicos, y de salud deben de desarrollar estrategias para la vinculación con la sociedad y el medio, no sólo transfiriendo conocimiento, sino escuchando, aprendiendo y reflexionando sobre el contenido de los mensajes de la comunidad, tal como se ilustra con la voz del actor social Docente 3

- "Nosotros llegábamos a la comunidad y la comunidad nos escuchaba a nosotros...ahora es la comunidad que nos dice a nosotros que quieren que nosotros digamos...ahora se tiene que pasar comunicación al consejo comunal y a una asamblea"

De esta manera se puede observar cómo la comunidad está empoderada de sus necesidades, o como dice el actor social Docente 2

- "de tecnologías de información"

y cómo la Universidad debe desarrollar nuevas estrategias para su vinculación con base en nuevas realidades emergentes.

Finalmente, se puede decir que a través de la asignatura enfermería comunitaria el estudiante desarrolla destrezas y habilidades en el área comunitaria, mejora su perfil, consolida y fortalece sus competencias, expone sus valores y se forma de manera integral. Es decir, se evidenció una interacción creadora entre Universidad y Comunidad, mediante la cual el quehacer en materia de salud se vincula con el fenómeno social a fin de producir las transformaciones necesarias para el logro de una mejor calidad de vida. 


\section{Significado de los Hallazgos para los Actores Sociales Estudiantes}

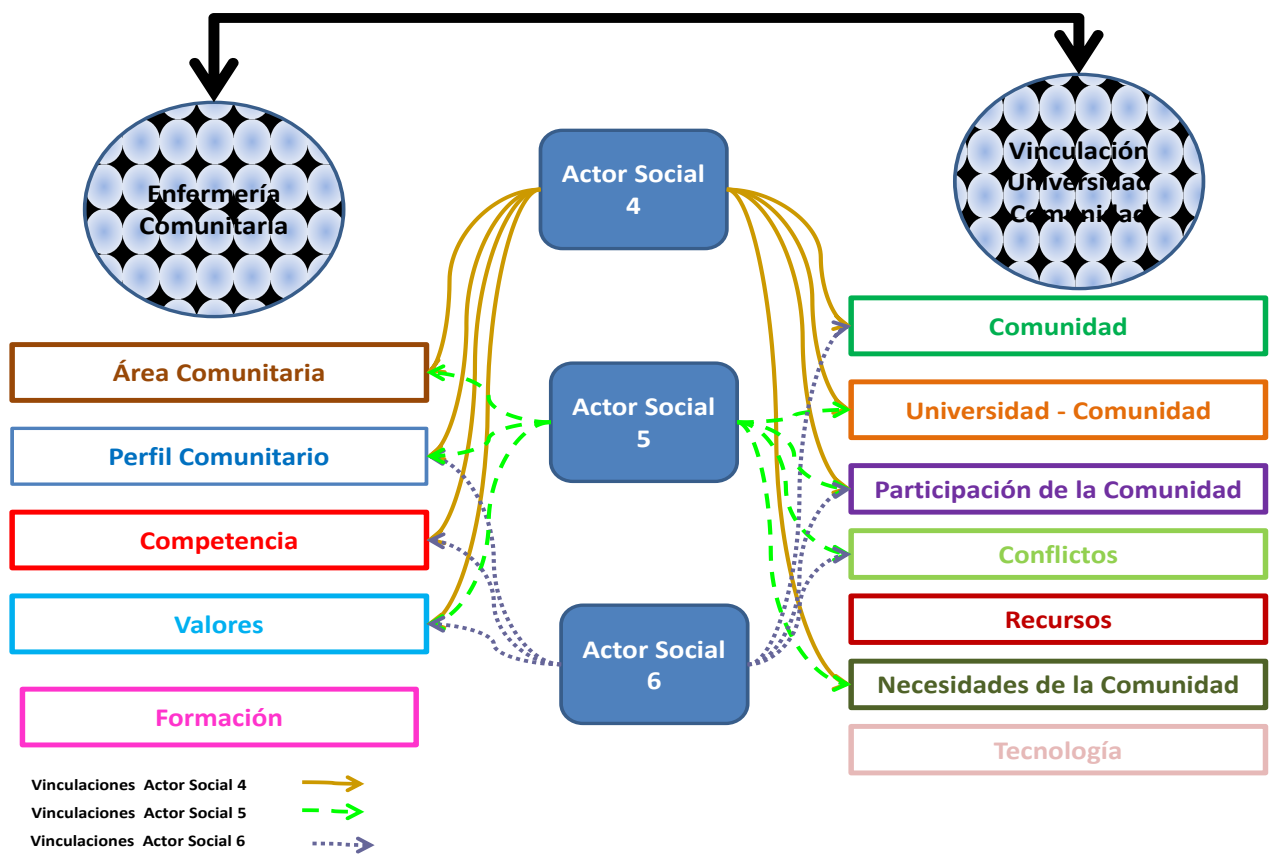

Infograma2: Vinculación de las Categorías de Análisis con las Subcategorías para los Actores Sociales 4, 5, 6 .

Se destaca de los hallazgos suministrados por los estudiantes, observados en el infograma 02, que los futuros licenciados en enfermería pueden a través de la asignatura enfermería comunitaria laborar en diversos ambientes, con personas, familias, grupos y la comunidad como un todo. De esta manera lo señala el actor social estudiante 4 al expresar

- "El trabajo en la comunidad tienes la oportunidad de estar en contacto directo con las personas, que de repente no es un problema de salud lo que los afecte, sino un problema familiar, enfermería somos de todo un poco"

Asimismo, se puede evidenciar que el estudiante desempeña un rol dinámico, pues éste cambia, a medida que suple las necesidades de la población. Entre las necesidades están la educación, promoción de la salud y la ejecución de programas de atención y prevención. Esto evidencia que el estudiante dentro de las actividades de Enfermería Comunitaria se transforma en un ente integrador de todos los conocimientos adquiridos en la Universidad, lo que le permite un rol dinámico en su participación comunitaria.

Se destaca que el estudiante de enfermería a través de la salud comunitaria valora la diversidad y complejidad de los factores que influyen sobre la salud de la comunidad. Para ello, realiza la valoración, lo cual posibilita la elaboración de diagnósticos comunitarios, éstos son útiles para determinar el enfoque específico según la planeación de las intervenciones de enfermería. Por lo tanto, el estudiante utiliza el proceso de cuidado en el desarrollo de sus actividades diarias, como son la visita domiciliaria, la educación para la salud, entre otras.

En tal sentido, los estudiantes que se entrevistaron trabajaron con estrategias dirigidas por los docentes y aceptadas por la comunidad. Sin embargo, alguno de 
ellos no tienen esa vocación de trabajar en la comunidad aunque sí en las acciones propias de la carrera como la prevención, diagnóstico, curación y rehabilitación de las enfermedades. Así lo expresa el actor social Estudiante 5

- "Eso de andar caminando, llevar sol, y a veces la gente no te atiende, andar atrás de ellos, esa parte no me agrada"

Se precisa de manera ineludible la participación activa de los estudiantes dentro de la comunidad con vocación de servicio comunitario. Algunos factores ambientales y sociales están inmersos dentro del trabajo social, lo que marca un significado propio en cada actor social en relación con su participación activa y significativa dentro de la comunidad.

Aunado a estas afirmaciones, la falta de recursos para el desarrollo de las prácticas comunitarias se revela como factor desmotivante en los estudiantes, así lo sostuvo el actor social estudiante 6:

- "Más que todo es eso, no tenemos las comodidades y los materiales necesarios"

Este factor puede favorecer de una manera negativa el desarrollo de las actividades comunitarias por parte de los estudiantes.

\section{Significado de los Hallazgos para los Actores Sociales de la Comunidad}

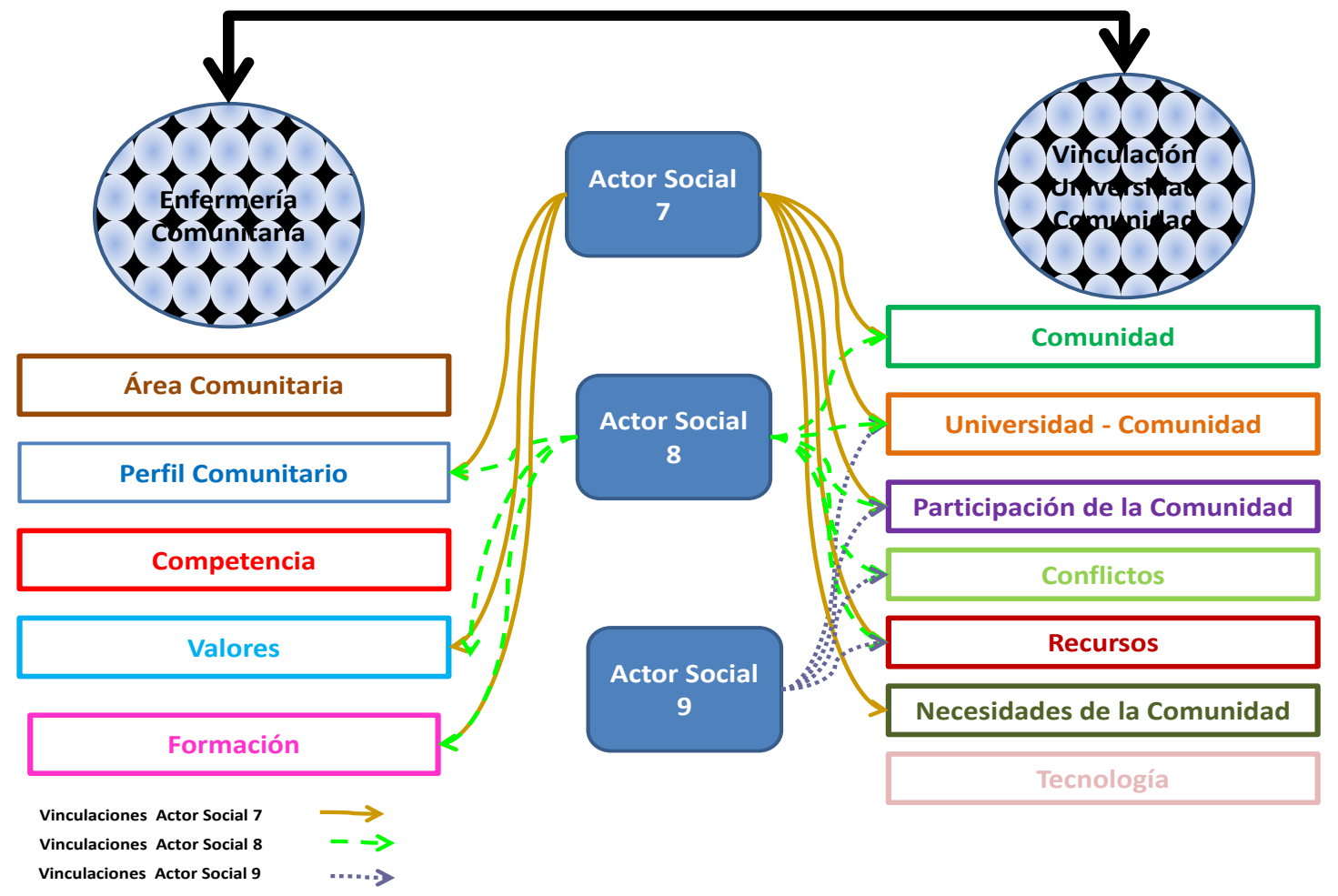

Infograma 3: Vinculación de las Categorías de Análisis con las Subcategorías de los Actores Sociales 7, 8, 9.

A través del infograma 3, se evidencia a través del significado de los hallazgos para los actores sociales de la Comunidad de las Tunas, que de manera general observaron el vínculo universidad-comunidad las siguientes afirmaciones: 
- La enfermería comunitaria, antes y ahora, se basa en la promoción de la salud y la prevención de la enfermedad utilizando para ello la educación como herramienta fundamental para obtener estilos de vida saludables en los grupos comunitarios, así lo mantuvo el actor social Comunitario 7

- "Bueno, son unos muchachos que legalmente se están preparando mucho, yo veo que ellos van y dan charlas a las comunidades".

Mediante esta afirmación se evidencia que la comunidad percibe los beneficios de la intervención comunitaria a través de los estudiantes.

- La formación académica en los estudiantes ha presentado cambios importantes a nivel universitario actual. En este sentido, el actor social comunitario 8 expresó:

"La formación es bastante bien, se ve que sí sabe lo que hacen. También veo que atacan las necesidades sentidas de la comunidad de hecho muchos han traído otras experiencias de otras comunidades y las aplican aquí"

De esta manera la propia comunidad le da significado a su propia experiencia al observar cambios significativos en el desarrollo académico de los estudiantes.

La práctica profesional permite un contacto con los actores de la comunidad en cuanto a horario, actividades y funciones. El actor social nuevo 9 sostuvo:

- "Por años hemos recibido la Universidad y hemos tenido unos avances muy importantes, lo que pasa es que el tiempo es muy reducido...ellos vienen solamente una vez a la semana...entonces realmente deberíamos abarcar más comunidades"

Según el significado del propio actor social, a pesar de poseer un contacto estrecho con la comunidad, el horario de prácticas comunitarias desarrollado por la Universidad no permite abarcar más actividades y funciones.

Es necesaria la reafirmación de la vocación de los estudiantes. Así lo expresó el actor social 9 -

"Cuando el muchacho llegue allá a escoger su carrera sea de verdad de corazón... hacer que el individuo se involucre en lo que verdaderamente quiere...para que luego puedan ayudar a los demás como de verdad tienen que hacerlo"

De esta manera la comunidad opina que un estudiante con vocación de servicio comunitario realizará de forma más eficiente sus actividades.

El estudiante está bien formado académicamente y le brinda de manera gratuita su formación a beneficio de la colectividad. Esta premisa es percibida por todos los actores sociales comunitarios, quienes no ponen en duda la capacidad profesional que tiene el estudiante en relación con sus conocimientos. El actor social Comunitario 7 manifestó:

- "Ellos dicen ahí vienen los muchachos de la UCLA y le abren las puertas, se ve que la gente está conforme con ellos" (C7, L38, L39). 
Esta expresión pone de manifiesto la satisfacción de la comunidad con las actividades de los estudiantes y, por ende, de su formación académica.

Falta de decisión y pérdida de liderazgo en las comunidades que ayuden al estudiante a la toma de decisiones. Esto lo sostuvo el actor social 9 al referir:

- "Entonces qué pasa que los líderes comunitarios no caminan las comunidades, como que no se interesan y más cuando estamos transmitiendo información sobre enfermedades de trasmisión sexual, embarazo precoz como que no les interesa mucho eso"

Dicho factor dificulta el desarrollo de las actividades comunitarias del estudiante, el significado que le imprime el actor social es por falta de liderazgo oportuno y motivador.

Así mismo, se debe reafirmar de valores como: empatía, fortaleza espiritual, vocación de servicio, confianza, educación y respeto. Al respecto el actor social Comunitario 9 manifestó que:

- "Cuando el muchacho llegue allá a escoger su carrera...se involucre en lo que verdaderamente quiere, primero que se identifique su entorno, que haga un diagnóstico de su familia, de su hogar, de su comunidad".

Para este actor involucrado en el estudio, los valores del estudiante nacen y se desarrollan dentro del seno de su familia y comunidad. Esta expresión del actor social Comunitario sin duda alguna constituyó un aporte fundamental al desarrollo de esta investigación al evidenciar la conciencia, por parte de los actores comunitarios, de que la educación y el respeto son parte de un conjunto de valores inmersos en el núcleo familiar.

La formación de los profesionales de enfermería debe ser con una visión holística que comprenda la salud y su recuperación como una situación de equilibrio armónico y dinámico entre la persona, el colectivo y el medio ambiente, ya que posee dimensiones sociales, psicológicas y biológicas como una consecuencia de la alteración de la integridad del ser humano. Esta afirmación evidencia, desde el significado que el propio actor comunitario le da a su contexto, que el estudiante debe formarse con una visión integral, que permanezca en el tiempo y le permita la gerencia de sus propios resultados, en el que el proceso salud enfermedad sea sistemático, armónico y sostenible, los cuales en ocasiones, por falta de continuidad, no se lleva a cabo.

El entramado de interacciones lógicas y formales que se articulan en la totalidad de la información proporcionada por los informantes claves indica que la lectura que los docentes de enfermería comunitaria hacen de la gestión académica, que adelantan y de los principios que manejan en un nivel de percepción teórica, alcanzan niveles integrales; los cuales, aunque no están teóricamente explicados a profundidad son advertidos con todo la potencialidad epistemológica que determinan. Los niveles teóricos del estado del arte del conocimiento que poseen los actores sociales entrevistados sobre los componentes del fenómeno educativo y comunitario que éstos concretan, aparece limitada en cuanto a su estructuración profunda y en ese sentido su contenido interno e intensivo es complementado con el carácter complejo que los 
informantes perciben en la expresión práctica, procedimental y orgánico funcional de la gestión que adelantan.

Lo anteriormente señalado lleva al investigador a evidenciar que, desde la visión de Husserl $^{6}$, y la cualidad fenomenológica de esta investigación; que el conocimiento que poseen los actores entrevistados sobre los componentes del fenómeno que concretan, se encuentra en el estadio superior de la percepción fenoménica como fase inicial de la aprehensión fenomenológica social y que en este momento, los actores sociales están entrando en la fase de reducción eidética del fenómeno que concretan, con la intención de fortalecer y consolida la gestión que adelantan.

La vinculación Universidad-Comunidad que manejaban los actores involucrados en torno al fenómeno que fue objeto de este estudio se constituyó en un modelo comunitario, que se encuentra en plena constitución teórico-conceptual y que contiene las fortalezas primordiales para la sustentación de una práctica de enfermería comunitaria, cuyo proceso se fundamenta claramente en el reconocimiento de la importancia de la gestión comunitaria, donde intervienen factores como el área comunitaria, el perfil comunitario, los valore implícitos en los actores sociales y la formación de dichos actores. Esto evidencia la posibilidad cierta de avance en cuanto al establecimiento de una gerencia comunitaria participativa, centrada en la vinculación Universidad-Comunidad en la cual intervienen por supuesto la comunidad, la participación de los actores de la comunidad, las necesidades que éstas poseen, los recursos con los cuales trabajan y los conflictos implícitos en un trabajo coordinado de los docentes, estudiantes y miembros de la comunidad en general.

Los docentes entrevistados reconocen la gestión académica y la importancia de vincular la universidad con la comunidad, se retroalimentan constantemente para originar procesos académicos y procedimientos de trabajo que apunta a la permanente expresión de niveles de acción educativa, cada vez más satisfactorios. Estos actores sociales, al hablar de su gestión como docentes dejan clara y coherentemente establecido que su acción discurre en el ámbito de una unidad holística, que interrelaciona profunda y dialécticamente los componentes del fenómeno en estudio.

Al observar el significado que le otorgan a su práctica académica, los docentes entrevistados coinciden en que su gestión responde a una sociología de servicio y gobernabilidad efectiva, en satisfacción de las exigencias que el entorno sociológico le hace a las instituciones que ellos gerencian. La disposición de los docentes entrevistados, en cuanto a la promoción y fortalecimiento del desempeño colectivo, como el resultado que trasciende la suma de los desempeños individuales está nucleada en torno a la promoción del estudiante y la consolidación de una cultura comunitaria que genere calidad de vida en el trabajo y desde la ejecución de las tareas que determinan los procesos académicos-laborales y comunitarios.

La práctica académica de los actores entrevistados está esencialmente influida por una filosofía organizacional comunitaria, que se centra en la vinculación universidadcomunidad y que los coloca ante la posibilidad de desarrollar conocimientos, saberes, habilidades y destrezas que les permitan ser un mejor estudiante-docente o servidor comunitario, así como un mejor ciudadano.

Como estrategia para el alcance de lo anteriormente señalado, los actores sociales del estudio sugieren que se debe tener primordial importancia en la formación 
permanente de los estudiantes y de los miembros de la comunidad para el ejercicio de sus funciones y que en este sentido, sus organizaciones valoran y ponen en prácticas estrategias educativas destinadas al fortalecimiento de la visión, la misión y la filosofía general de la organización. Llama la atención, que los actores entrevistados coinciden en que ellos valoran y controlan su gestión (académica y comunitaria) desde la integración unitaria de los resultados que gerencian y alcanzan, en la atención de las necesidades que el entorno social le hace a las instituciones, centrándose en la naturaleza de la organización que lideran.

Los significantes y significados que los actores entrevistados le asignan a los principios epistemológicos que sirven de anclaje a su práctica gerencial se integran en una concepción que define a los conocimientos y saberes que necesita la universidad y la comunidad, como el resultado de procesos cognitivos que emergen en la acción colectiva y que sólo pueden ser desarrollados, consolidados y extendidos en el contexto institucional enmarcado en la comunidad, a través de la participación abierta, interactiva, dinámica y auto reflexiva de todos y cada uno de los actores sociales.

\section{Reflexiones de Cierre}

Existe integración y calidad en la relación Universidad - Comunidad con los procesos sociales, productivos, económicos, y de salud a través del desarrollo de estrategias para la vinculación con la sociedad y el medio, no sólo transfiriendo conocimiento, sino escuchando, aprendiendo y reflexionando sobre el contenido de los mensajes de la comunidad. Se logra describir el vínculo Universidad - Comunidad para el actor social Docente desde la Cátedra Enfermería Comunitaria.

Por otro lado, a través de la interacción dialógica con el Docente, los significados de algunos factores que inciden en el vínculo Universidad - Comunidad siempre fue abordado de una forma positiva. El docente afirma que la Universidad provee de todo lo necesario, el cual contrastó con lo expresado por los estudiantes.

Así mismo, existe una clara y completa definición de lo que es la enfermería comunitaria, que conocen perfectamente hacia donde se dirigen los estudiantes y qué se espera de ellos. De esta manera se constató el significado que el docente le otorga a la relación Universidad, Docente, Estudiante y Comunidad

Por otro lado, se obtuvo como resultado que el estudiante desempeña un rol dinámico, pues cambia, a medida que suple las necesidades de la población. Entre las necesidades están la educación, promoción de la salud y la ejecución de programas de atención y prevención. Es así, como se describe el vínculo Universidad - Comunidad desde la Cátedra Enfermería Comunitaria que el estudiante le asigna a su propia experiencia.

Se logró evidenciar, que los estudiantes trabajaron con estrategias dirigidas por los docentes y aceptadas por la comunidad. Sin embargo, muchos de ellos no tienen esa vocación de trabajar en la comunidad. Aunque sí en las acciones propias de la carrera como la prevención, diagnóstico, curación y rehabilitación de las enfermedades. Esto logró establecer uno de los factores que inciden en el vínculo Universidad Comunidad. El investigador asume, por lo tanto, que uno del obstáculo que encuentra la enfermería comunitaria como profesión es, la actitud de los estudiantes, la apatía y el manejo de conflicto, ya que por un lado intentan superar los miedos en un área comunitaria desconocida y por la otra lograr establecer su práctica profesional con 
base en fundamentos de la Salud Pública y Comunitaria. No obstante los estudiantes cuentan con la preparación académica que le permite asumir estos retos.

Entre tanto, para la comunidad, la enfermería comunitaria se basa en la promoción de la salud y la prevención de la enfermedad utilizando para ello la educación como herramienta fundamental para obtener estilos de vida saludables en los grupos comunitarios. Se evidenció que la comunidad percibe los beneficios de la intervención comunitaria a través de los estudiantes lo que describe el vínculo Universidad Comunidad desde la Cátedra Enfermería Comunitaria desde su propia perspectiva.

Por otra parte, la comunidad afirmó que es necesaria la reafirmación de la vocación de los estudiantes. Desde esta perspectiva se observa que la comunidad describe uno de los factores que inciden en el vínculo Universidad-Comunidad. Este factor dificulta el desarrollo de las actividades comunitarias del estudiante, el significado que le imprime el actor social es por falta de liderazgo oportuno y motivador.

No obstante, el estudiante está bien formado académicamente y le brinda de manera gratuita su formación a beneficio de la colectividad. Esta premisa es percibida por todos los actores sociales comunitarios, quienes no ponen en duda la capacidad profesional que tiene el estudiante con base en sus conocimientos. Se pone de manifiesto la satisfacción de la comunidad con las actividades de los estudiantes y, por ende, la relación Universidad, Docente, Estudiante, Comunidad, logrando uno de los objetivos de este estudio.

\section{REFERENCIAS}

1.Watson, D. y otros. Managing Civic and Community Engagement. McGraw-Hill International. Traducido por Martínez J. 2007.

2.Hall, L. The Funding and Development of Community-University Research Partnerships in Canada. Ottawa: SSHRC. Traducido por Martinez J. 2009.

3.Rivas Y., y otros. La Universidad venezolana. Barquisimeto - Lara: Universidad Nacional Experimental Simón Rodríguez - Convenio Alcaldía Iribarren. 2008.

4. Ley del Servicio Comunitario. Gaceta Oficial № 38.272 del 14 de septiembre la figura del servicio comunitario. 2005.

5.Morse. J. Asuntos críticos en los métodos de investigación cualitativa. Facultad de enfermaría de la Universidad de Antioquia. Contus. 2003.

6. Husserl, E. Ideas relativas a una fenomenología pura y a una filosofía fenomenológica. 3a. edición. México: F.C.E. 1989.

7.Ruiz O. Metodología de la investigación cualitativa. Universidad de Deusto. Bilbao. 1996.

8.Valles, M.S. Entrevistas cualitativas, Madrid: CIS (Colección Cuadernos Metodológicos, n. ㅇ 32). 2007.

9.Paris, M.J. Investigación cualitativa en salud: superando los horrores metodológicos. Salud, Cambio social y política. 1997.

10. Lincoln, Y. Conexiones afines entre los métodos cualitativos y la investigación en salud. Investigación y Educación en Enfermería 15(2):57-70. 1997.

11. Eyssautier de la Mora, M. Metodología de la investigación: desarrollo de la inteligencia. Mexico. Thomson. 2006.

12. Lincoln, Y, y Guba, E. Naturalistic inquiry. New York: Sage. 1985.

13. Rodríguez, G., Gil, J. y García, E. Metodología de la Investigación Cualitativa. Segunda Edición. Ediciones Aljibe. 1999. 
14. Strauss, A y Corbin J. Bases de la Investigación Cualitativa. Editorial Universidad de Antioquia. 2002.

15. González, G. y Hernández, T. Interpretación de la evidencia cualitativa. 1era edición. Barquisimeto - Venezuela: Gema. 2011.

16. Bustingorry, S., Sanchez, I. y Ibañez, F. Investigación Cualitativa en Educación hacia la Gerencia de la Teoría a través del Proceso Analítico: Ensayo. 2006.

17. Ramírez, M. Los estudios comunitarios desde una perspectiva espacial. Documento en línea disponible en http://www.uh.cu. 2005.

18. Almaguer, R. Democracia y localidad. En: Vázquez, A. y Dávalos, R. (compiladores). Participación social. Desarrollo urbano y comunitario. Universidad de La Habana. 1996. 